\title{
Special issue on knowledge integration and management in autonomous systems
}

It is important that software that operates over modern computer infrastructure adopt accordingly, to provide highly customised services to everyone who needs them. New requirements have to be met, for example the systems must still evolve and integrate many heterogeneous systems. Furthermore, the systems must cope with changes in network topology, node failures, collaborations and cooperation between different types of customized knowledge. As information systems get more complex, it becomes more difficult to integrate those using traditional approaches. Over recent years there has been a significant increase in interest in autonomous, self-organisation applications that undertake problem solving based on distributed interactions between uncoupled components, i.e. SOA, MAS. Many issues such as fuzzy, nature-inspired methodologies and hybridisation of these methods still remain open.

The aim of this special issue is to highlight an on-going research on different methodological and technological approaches of self-organization and knowledge integration together with their applications on various domains. The papers cover some of the most important aspects of knowledge integration and management in autonomous systems and can serve as a reference point for this exciting area. In particular, they describe automated multi-issue bilateral negotiations and negotiation protocols, ontology mapping composition for query transformation, constructing and mining a semantic-based academic social network, solar cell monitoring and how swarm-based multi-agent systems cab be designed using MaSE methodology.

The papers in this issue have been reviewed by prominent experts in this field. As the result of thorough review process, we have selected six papers for final publication.
The first paper by Lopez-Carmona et al. titled "Improving trade-offs in automated bilateral negotiations for expressive and inexpressive scenarios" presents three new mechanisms to improve the similarity-based trade-off algorithm for win-win solutions. Two of them are applicable in expressive scenarios, where agents may be willing to share preference information, while the third one is intended for inexpressive scenarios. To validate their hypotheses and evaluate the effects of their contributions, they have performed single shot experiments, testing isolated runs of the proposed mechanisms.

The following paper, titled "Secure and efficient protocols for multiple interdependent issues negotiation", by Fujita, Ito and Klein also concerns an important problem in multi-issue negotiation in which agent utility functions are nonlinear. The authors propose Distributed Mediator Protocol and Take it or Leave it Protocol for negotiation that can reach agreements and completely conceal agents' private information. Moreover, they propose Hybrid Secure Protocol that combines Distributed Mediator Protocol with Take it or Leave it Protocol and achieves high optimality and uses less communication cost in both cone-constraints and cube-constraints situations.

The third paper by Jung titled "An empirical study on optimizing query transformation on semantic peerto-peer networks" analyses how to make the existing mapping information sharable and exchangeable. It means that we can collect the existing mapping information and aggregate them. Consequently, we can estimate the ontology mappings in an indirect manner. To evaluate the performance of sharing and composing mapping results, a multi-agent platform has been employed. Any two heterogeneous agents can communicate with each other by query-answering process, 
so that they can measure how precisely the proposed mapping composition is conducted.

The article titled "Constructing and Mining a Semantic-based Academic Social Network" by Duong et al. included in the fourth position of this issue a novel method for building an academic social network based explicitly on researchers' knowledge interests is considered. Mining in the direct loop graph-based social network has been explored for two problems, Expert Ranking and Expert Finding. They defined a dataset (www.kmnets.info) for evaluation of their proposal. The results show that a social network based on profile matching is more representative than network based on publication co-authorship or shared keywords.

In "Applying KeyGraph and Data Crystallization to Technology Monitoring on Solar Cell", Chiu describes how solar cell monitoring is helpful for companies, employees, and stakeholders to understand the technological situation of their supplying products. Based on the technology monitoring, the relations between companies and subtopics, between companies and applications, and among companies were observed and the suggested strategies for some specific companies were proposed so as to provide an external viewpoint for the decision makers to facilitate their strategic planning. In addition, an evaluation has been carried out in order to examine the validity of the study.

Finally, in the sixth paper titled "Use of MaSE methodology for designing a swarm-based multiagent system", Król and Drożdżowski study how the Multiagent Systems Engineering methodology can be used to bridge the gap between swarm intelligence mechanisms and multi-agent systems. The paper offers how the intersection between two most common metaheuristics from swarm intelligence: ant colony optimisation, particle swarm optimisation, and multi-agent systems opens new significant and highly promising possibilities. During the test, full functionality and scalability of swarm-based multi-agent systems designed using MaSE methodology have been confirmed.

In concluding, we would like to thank all those that contributed to this special issue. First of all, we would like to thank Prof. Reza Langari, Editor-in-Chief of Journal of Intelligent \& Fuzzy Systems for giving us the opportunity to organise this special issue. Special thanks go to the reviewers for their thorough comments that help in enhancing the quality of the papers. Last, but not least, we thank all the authors who submitted papers to this special issue.

\section{Short bios of guest editors}

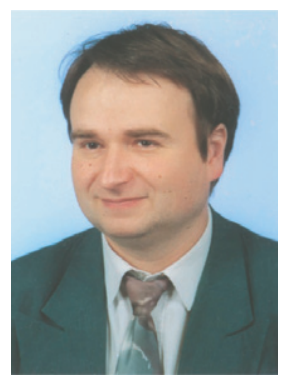

Dariusz Król, Ph.D.: Assistant professor in Institute of Informatics at Wrocław University of Technology in Poland. Currently working on knowledge evolution and integration, multi-agent systems and grid computing, computational intelligence, adaptive and self-organising systems. Co-editor of 1 book, author of 2 academic books and over 80 other publications including book chapters, journal and conference papers. He is an Action Editor of Computational Intelligence magazine (Wiley-Blackwell), Associate Editor of International Journal of Distributed Systems and Technologies (IGI Global) and Editor of International Journal of Information Technology, Communications and Convergence. He has served on programme committees for a number of international conferences in the areas of distributed and grid computing, Web technologies, intelligent and multi-agent systems, like. He was rewarded by IBM for the work on Eclipse Didactic Distribution and by DAAD for the project on Structural Performance Evaluation for Knowledge Integration.

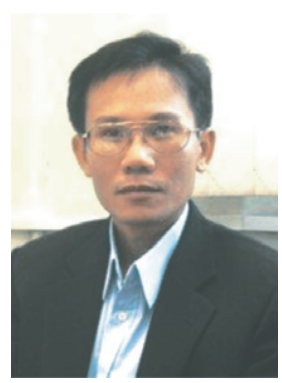

Professor Ngoc Thanh Nguyen, Ph.D., D.Sc., currently works at Wroclaw University of Technology, Poland, and is the head of Knowledge Management Systems Department in the Faculty of Computer Science. He is the author or editor of 11 books and about 200 other publications. His latest monograph entitled "Advanced Methods for Inconsistent Knowledge Management" has been published by Springer in 2008. He is the Editor-in-Chief of two international journals: "LNCS Transactions on Computational Collective Intelligence" and "International Journal of Intelligent Information and Database Systems"; Editor-in-Chief of 2 book series for IGI Global Publisher (USA). He is the Associate Editor of 4 prestigious international journals and a member of Editorial Boards of several other prestigious international journals. He is the founder of Asian Conference on Intelligent Information and Database Systems (ACIIDS). Besides he is also the founder of 2 other conference series on Collective Intelligence and Multi-agent Systems held in Europe and Asia-Pacific. Prof. Nguyen Ngoc Thanh has been selected and honored as the Vice-President of International Society of Applied Intelligence (USA); Senior Member of IEEE and ACM. He is also an expert of European Commission in evaluation research projects and an expert of Polish Ministry of Science and Higher Education and Slovakia Research Agency. He is also the Associate Chair of KES society (UK) and many other functions in international societies like IFIP, WIC etc. In 2009 Prof. Nguyen has been nominated by ACM of title "Distinguished Scientist".

Institute of Informatics, Wroctaw University of Technology, Wyb. Wyspiańskiego 27, 50-370

Wroclaw, Poland

E-mail: dariusz.krol@pwr.wroc.pl (D. Król), thanh@pwr.wroc.pl (N.T. Nguyen). 